Int. J. Odontostomat., 9(1):43-52, 2015.

\title{
Odontología Basada en Evidencia: Las 82 Revistas de Mayor Impacto
}

\author{
Evidence Based Dentistry: The 82 Highest Impact Journals
}

\author{
Marco Alarcón*; Christian Aquino*; César Quintanilla*; Lucy Raymundo* \& Javier Álvarez*
}

ALARCÓN M.; AQUINO, C.; QUINTANILLA, C.; RAYMUNDO, L. \& ÁLVAREZ, J. Odontología basada en evidencia: Las 82 revistas de mayor impacto. Int. J. Odontostomat., 9(1):43-52, 2015.

RESUMEN: La Odontología Basada en Evidencia es la aplicación clínica de las mejores evidencias científicas basadas en la experiencia del operador y en las expectativas del paciente. Estas evidencias científicas se presentan día a día en revistas indexadas alrededor del mundo con una actualización constante. El objetivo de la presente revisión es presentar y describir las características de las revistas de mayor impacto científico en el área de Odontología sirviendo como base para la mejor toma de decisiones clínicas en nuestros pacientes. La búsqueda de la información se realizó según el Indice de factor de impacto del ISI Web of Science 2014.

PALABRAS CLAVE: factor de Impacto, factor de Impacto de la revista, portales de acceso a revistas científicas, Medline, artículo de revista.

\section{INTRODUCCIÓN}

La necesidad de una odontología predecible y exitosa ha llevado a un cambio en el paradigma de atención tradicional hacia la Odontológica Basada en Evidencia (OBE). El primer nivel dentro de ése concepto es la búsqueda de la mejor evidencia científica para la toma de decisiones en nuestros pacientes (Feijoo et al., 2014).

La calidad y la repercusión de la actividad científica se miden hoy en día en el factor de impacto de las revistas científicas. El factor de impacto es una medida de la frecuencia en la cual un artículo de una revista ha sido citado en un periodo de tiempo (Jalalzadeh \& Sangari, 2011; Oosthuizen \& Fenton, 2014). Cada año el Institute for Scientific Information (ISI) calcula la repercusión de las revistas científicas, las cuales son publicadas en un informe llamado Journal Citation Report (JCR).

El propósito del presente artículo es identificar las revistas odontológicas indexadas de mayor impacto a nivel mundial y analizar sus características las cua- les nos ayudarán a tomar las mejores decisiones clínicas basadas en la mejor evidencia científica.

Antiguamente, el acto de publicar investigaciones era discutido e incluso menospreciado. No era común que un nuevo descubrimiento se anunciara como un artículo, desconociendo la prioridad del descubridor, siendo indescifrable la evidencia de la autoría de cualquier investigación. Entre las primeras publicaciones científicas se encuentran el Journal des Scavans, publicado en París (1665), la Philosophical Transactions of the Royal Society (Inglaterra) y la Acta Eruditorum (Leipzig, Otto Mencke), ambas a finales del siglo XVII (Garfield, 2006).

La ISI Web of Science es un servicio en línea de información científica, suministrado por el ISI, grupo integrado en Thomson Reuters. Esta web facilita el acceso a un conjunto de bases de datos bibliográficas y otros recursos que abarcan todos los campos del conocimiento académico, permitiendo descubrir el impacto de un trabajo científico sobre la investigación

*Posgrado en Estomatología. Universidad Peruana Cayetano Heredia, Lima, Perú. 
actual y por último, permite conectarse al texto completo de publicaciones primarias y otros recursos accediendo a ellos mediante un sistema de búsqueda basado en palabras clave (Yaminfirooz et al., 2014; Brown, 2011).

Factor de impacto. El factor de impacto $(\mathrm{FI})$ de una revista es la media del número de veces que se cita un artículo publicado en una revista determinada. Es un instrumento para comparar revistas y evaluar la importancia de una revista específica dentro de un mismo campo científico. Este indicador (FI) es calculado como el número de citaciones en documentos publicados en una revista científica durante dos años (Ci), dividido por el número de artículos (Ar) publicados por esa revista en el mismo período de tiempo (Owlia et al., 2011; Gonzalez-Pereira et al., 2010; Falagas et al., 2008; Kulasegarah \& Fenton, 2010).

\section{Utilizando la siguiente fórmula: $\mathrm{Fi}=\mathrm{Cl} / \mathrm{Ar}$}

El FI es calculado anualmente por el Institute for ISI Web of Science de las revistas incluidas en el Journal Citation Report (Chang et al., 2013).

El Eigenfactor Score. Otra manera de evaluar las publicaciones de mayor impacto es mediante el indicador Eigenfactor, que fue desarrollado por Jevin West y Carl Bergstrom de la Universidad de Washington cuya diferencia con el FI radica en que el Eigenfactor Score da prioridad a aquellas citas que proceden de revistas influyentes. Como consecuencia, estas revistas ejercen una mayor influencia en la resolución de la clasificación de las revistas a las que hacen referencia. El índice Eigenfactor Score no tiene en cuenta las autocitas, siendo más ajustado a la realidad (Kianifar et al., 2014; Sillet et al., 2012; Berhidi et al., 2009; Fardi et al., 2011).

\section{MATERIAL Y MÉTODO}

Se realizó una búsqueda electrónica en el Institute for Scientific Information (ISI) Web of Science de las revistas incluidas en el Journal Citation Report (edición Setiembre 2014) en la categoría de "Odontología". Cada una de las 82 revistas seleccionadas fue analizada utilizando la herramienta de búsqueda "Impact Factor" de la base de datos Web of Science para identificar las revistas con más impacto a nivel mundial. La información recopilada nos proporcionó el nombre de la revista, país de origen/editorial y área de investigación.

\section{RESULTADOS}

Revistas indexadas en Odontología. De un total de 82 revistas científicas indexadas en Odontología disponibles en el Journal Citation Reports (JCR) de la base de datos ISI Web of Science, la revista con mayor impacto científico es el Dental Materials $(4,160$ de $\mathrm{FI})$, seguidas por el Journal of Dental Research $(4,144)$, Journal of Clinical Periodontology $(3,610)$. Completando las 10 revistas más importantes el Clinical Oral Implants Research $(3,123)$, Oral Oncology $(3,029)$, Periontology 2000 (3,000), Molecular Oral Microbiology $(2,841)$, Journal of Dentistry $(2,840)$, Clinical Implant Dentistry and Related Research $(2,796)$, Journal of Endodontics (2,788). La lista completa de revistas indexadas en odontología es presentada en la Tabla I.

Teniendo en cuenta el Eigen factor las 3 revistas con mayor puntaje son: Journal of Dental Research $(0,02235)$, seguidas por el Journal of Ora and Maxillofacial Surgery $(0,01822)$, Oral Surgery Oral Medicine Oral Pathology Oral Radiology $(0,01615)$. La lista completa de revistas indexadas en odontología es presentada en la Figura 1.

La productividad científica medida según especialidades tuvo un predominio multidisciplinario siendo la Odontología General (22 revistas) la que ocupó el primer lugar seguido por el área de Cirugía Oral (12 revistas), Implantología (6 revistas), Ortodoncia (6 revistas), Periodoncia (5 revistas), Rehabilitación Oral (5 revistas), Patología oral (3 revistas). La lista completa de revistas indexadas en odontología es presentada en la Figura 2.

Otro tema para analizar es la distribución demográfica del país de origen de las publicaciones científicas, encontrándose que la mayoría de las publicaciones se efectuaron en Estados Unidos (27 revistas), seguidamente Dinamarca (17 revistas), Inglaterra (15), Alemania y Japón (3 revistas respectivamente). La lista completa de revistas indexadas en odontología es presentada en la Figura 3.

Otro aspecto considerado en la presente revisión es la que corresponde a las editoriales entre las que destacaron: WILEY-BLACKWELL (24 revistas), ELSEVIER SCI LTD (8 revistas), QUINTESSENCE PUBLISHING CO INC (8 revistas), CHURCHILL LIVINGSTONE (3 revistas). La lista completa de revistas indexadas en odontología es presentada en la Figura 4. 


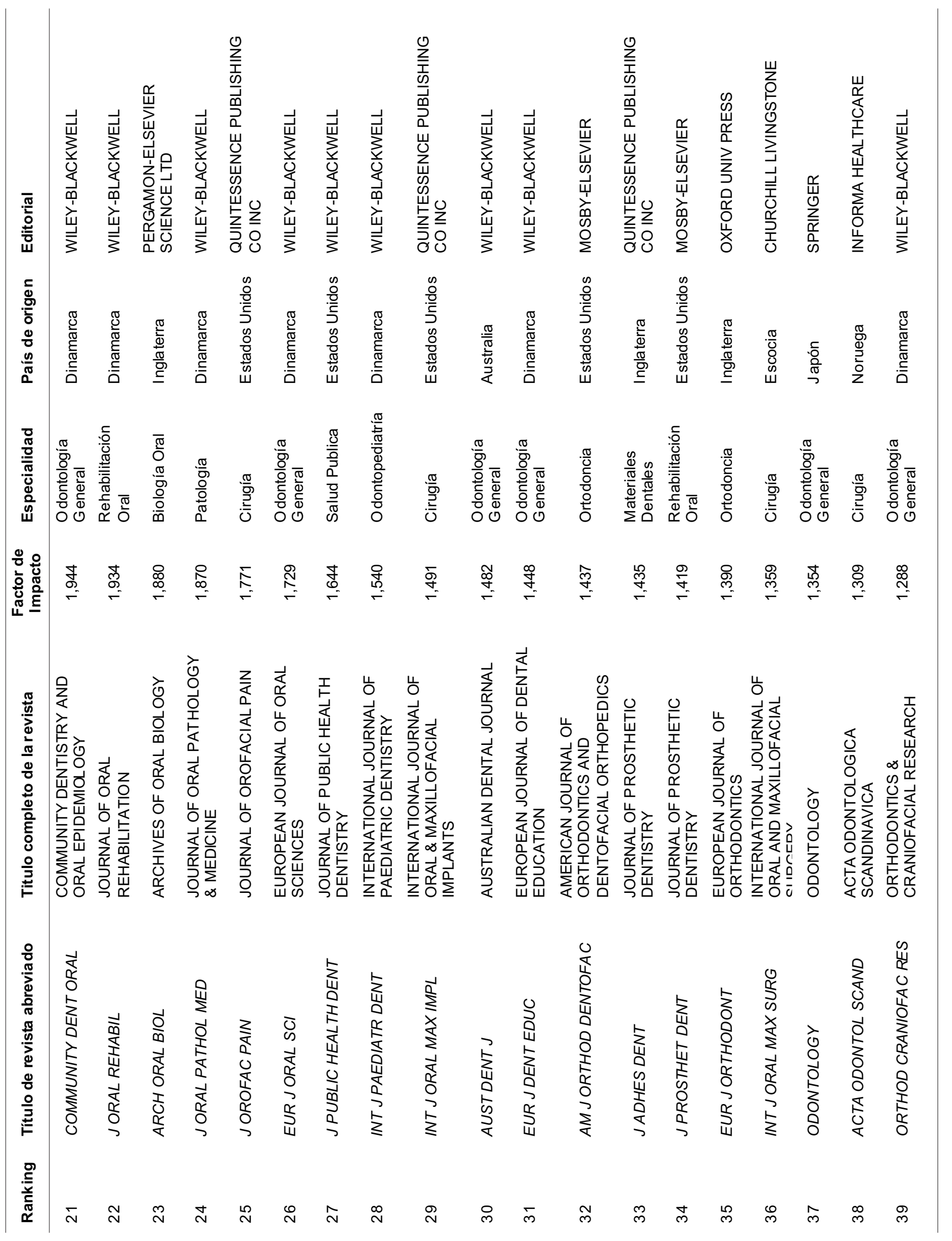




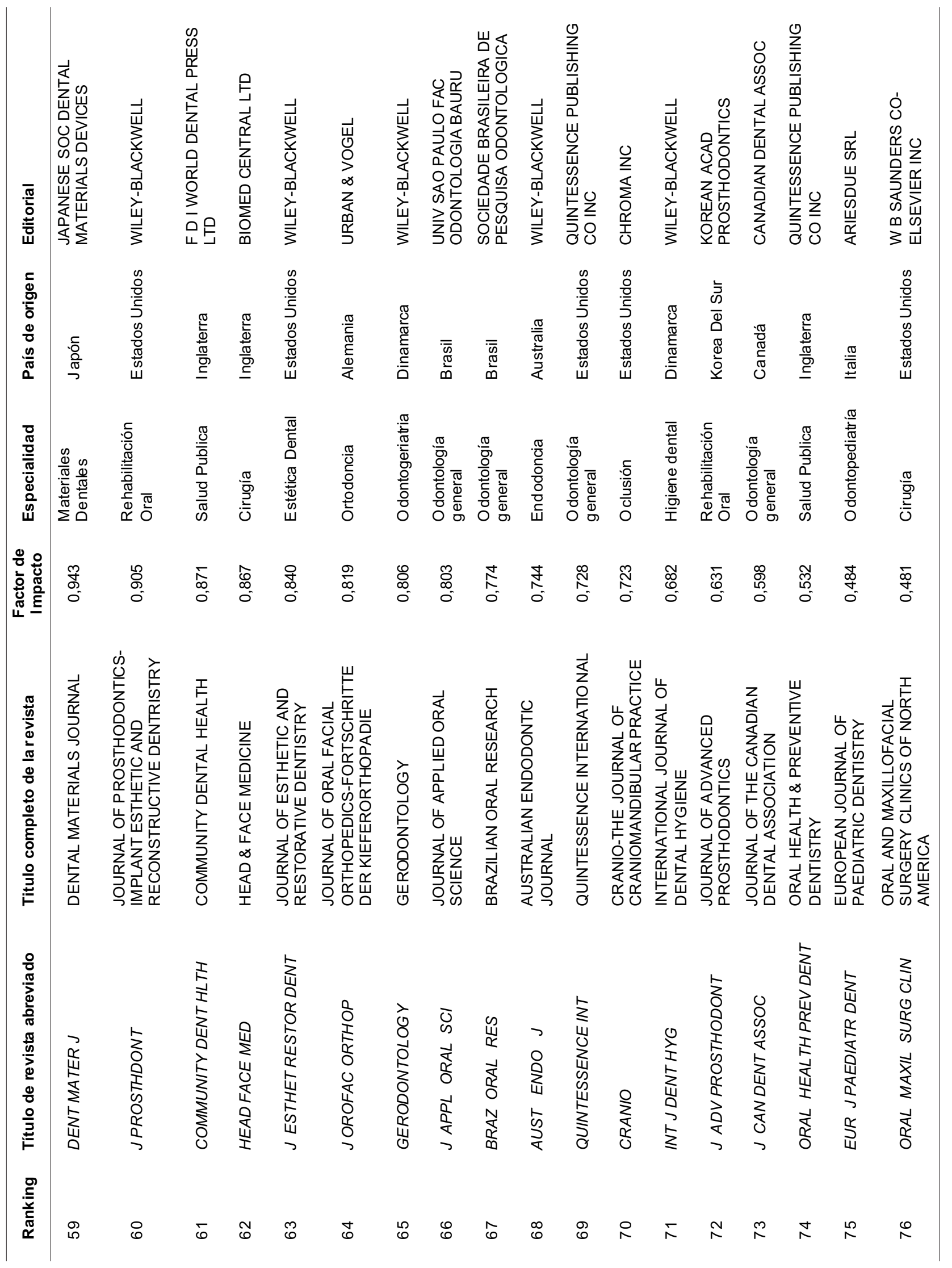



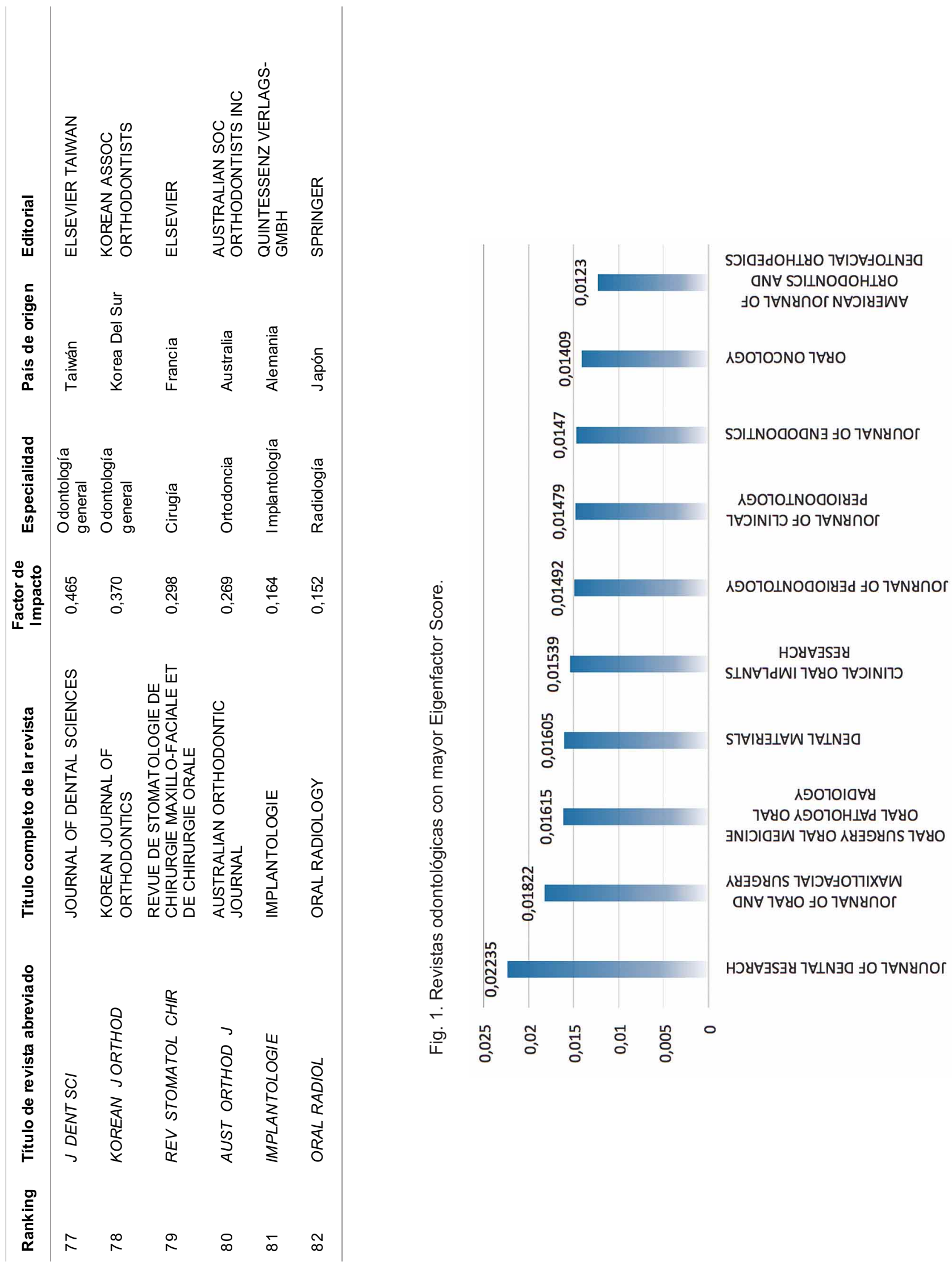
ALARCón M.; AQUINO, C.; QUINTANILLA, C.; RAYMUNDO, L. \& ÁLVAREZ, J. Odontología basada en evidencia: Las 82 revistas de mayor impacto

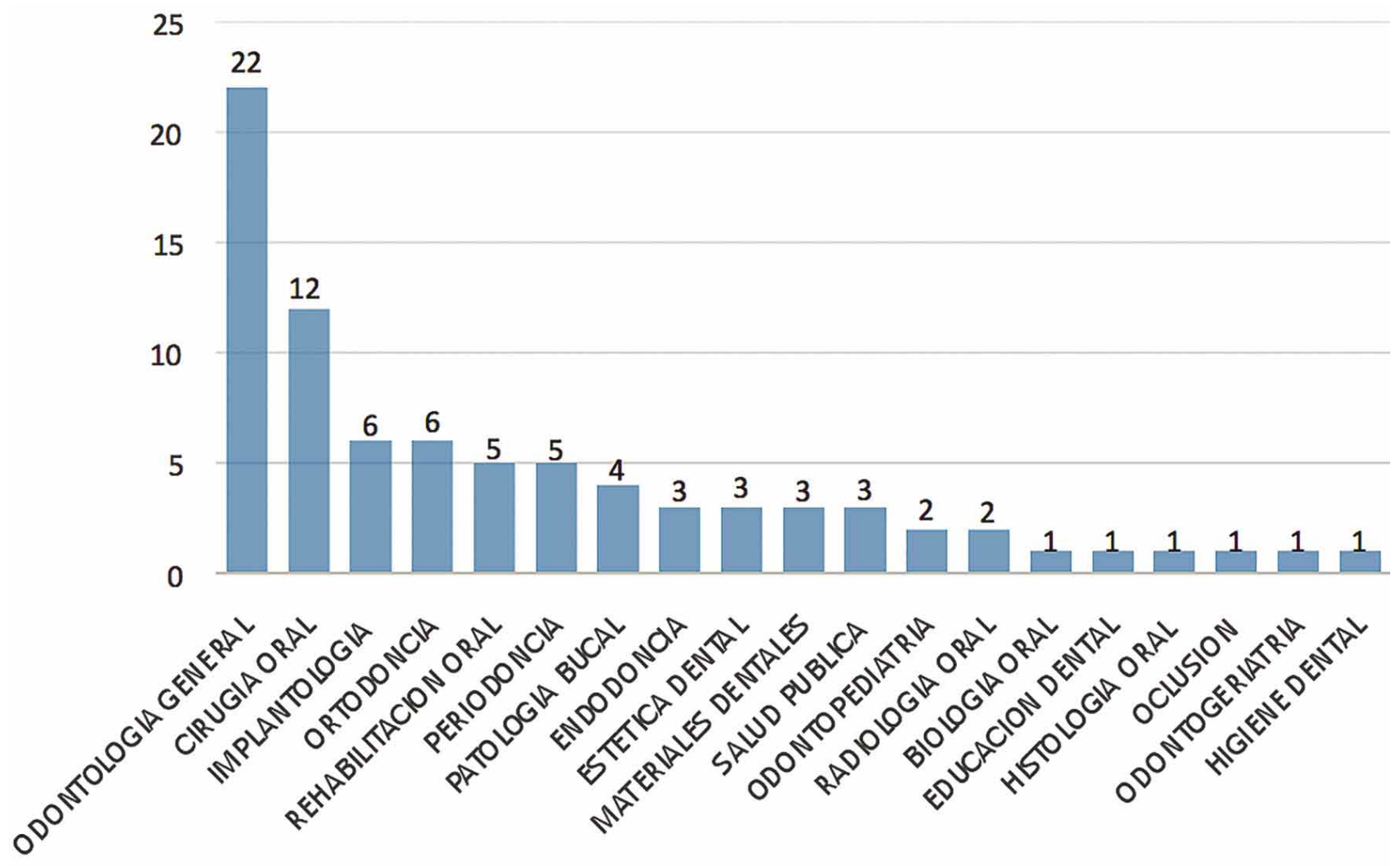

Fig. 2. Revistas científicas de mayor impacto en odontología por especialidad.

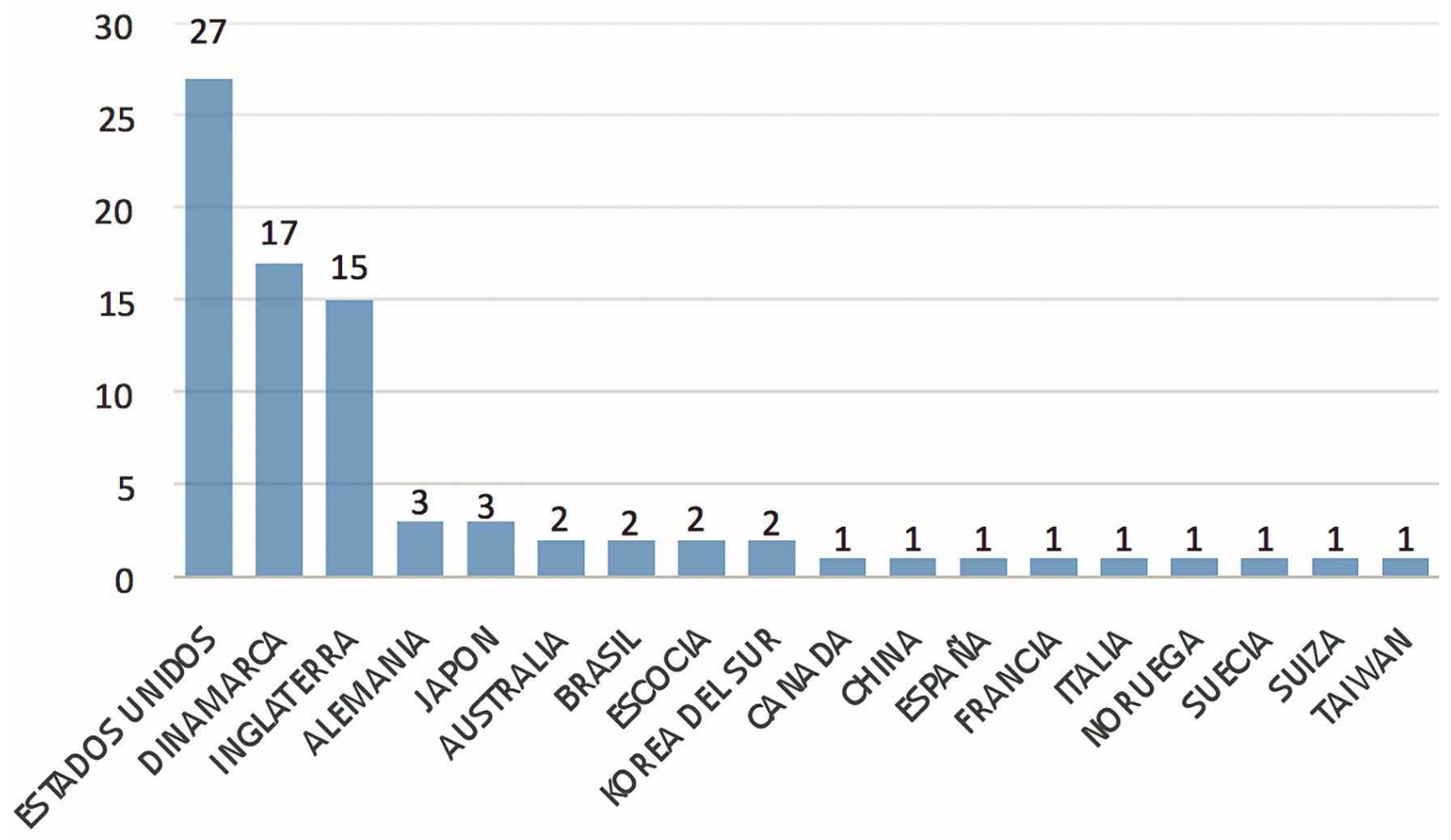

Fig. 3. Revistas científicas de mayor impacto en odontología según país de origen. 


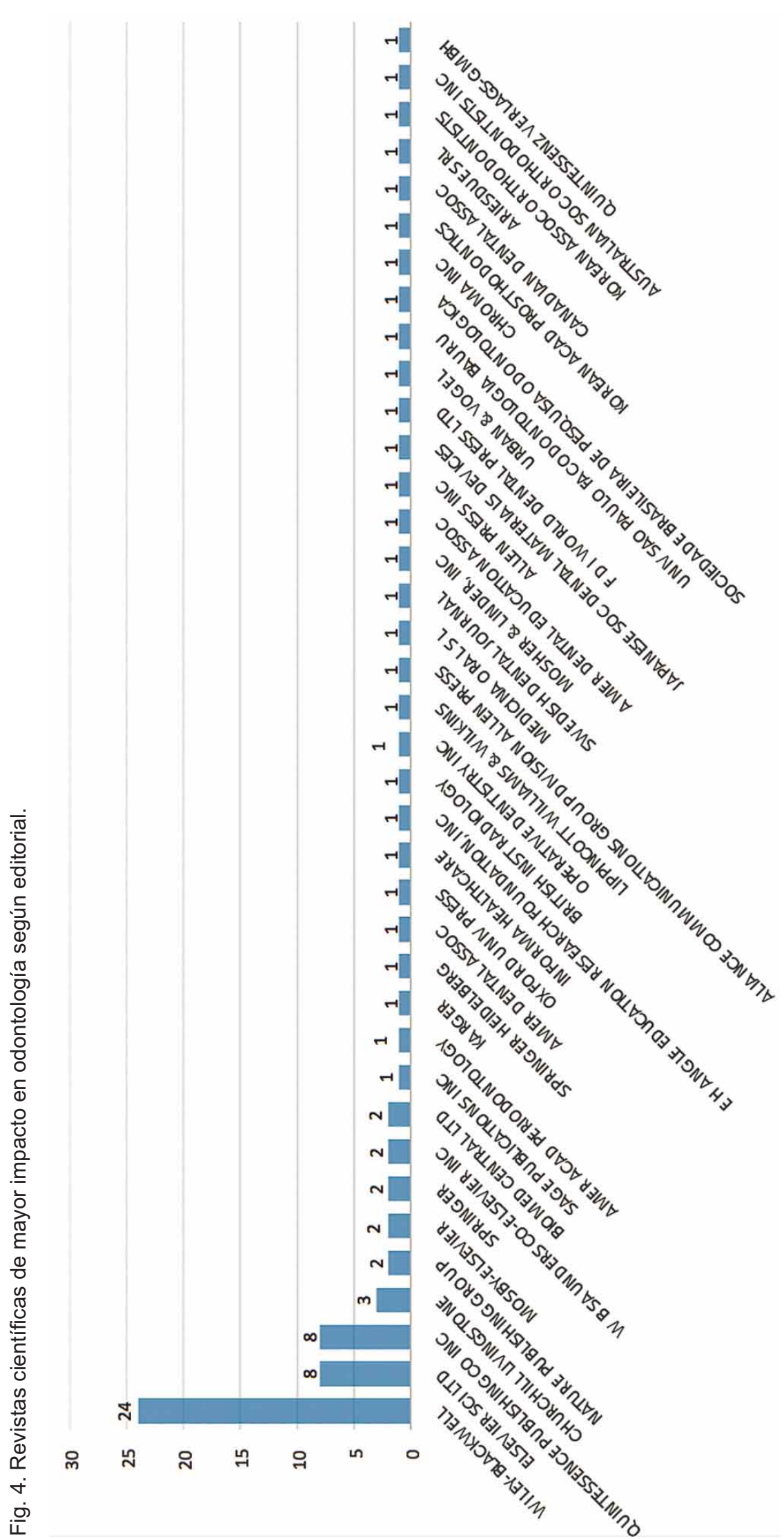

\section{DISCUSIÓN}

La investigación científica en Odontología, puede ser cuantificada en forma individual y colectiva, siendo el FI una herramienta de medición válida del nivel de las publicaciones científicas. Independientemente de las limitaciones que todo índice de impacto presenta, los datos recolectados demuestran que no se obtiene una producción óptima; evidenciándose esto en el FI del JOURNAL DENTAL MATERIALS $(4,160)$, es muy bajo comparado a otras áreas biomédicas. Se deben considerar los factores adversos que influyen en la baja productividad realizando esfuerzos para lograr aumentar los índices cuantitativos y cualitativos ya que la evidencia confiable repercute en el desarrollo social de la investigación en salud a nivel mundial.

Al comparar el FI y el Eigenfactor Score, las revistas con mayor puntaje difieren siendo respectivamente el Journal Dental Materials y Journal of Dental Research. Esta variación puede interpretarse debido a que el nuevo índice Eigenfactor Score busca ser más preciso debido a que excluye las autocitas y considera como referencia las revistas más influyentes (Ramin \& Sarraf Shirazi, 2012).

A la evaluación de las diez primeras revistas según el FI podemos apreciar una diversidad entre las áreas odontológicas resaltando revistas que desarrollan temas relacionadas a Materiales Dentales, Periodoncia, Implantologia y Cáncer Oral. Estos temas van de acuerdo a la actualidad odontológica que 
van desarrollando líneas de investigación en estas áreas debido al poco conocimiento y desarrollo científico y tecnológico. Es interesante saber que las áreas de Rehabilitación, Ortodoncia han sido desplazadas a un segundo nivel.

Debemos resaltar la calidad científica de países como Dinamarca, Inglaterra y Japón, estos van acorde con su desarrollo y sus alcances en tecnolo- gía, las revistas europeas tienen uno de los mayores índices de impacto con una población menor que los Estados Unidos.

La búsqueda y selección de un adecuado artículo científico, en una buena revista indexada y con alto factor de impacto es parte fundamental del desarrollo de la Odontología Basada en Evidencia la cual todo profesional debería conocer.

ALARCÓN, M.; AQUINO, C.; QUINTANILLA, C.; RAYMUNDO, L. \& ÁLVAREZ, J. Evidence based Dentistry: The 82 highest impact Journals. Int. J. Odontostomat., 9(1):43-52, 2015.

ABSTRACT: Evidence-Based Dentistry is the clinical application of the best scientific evidence based on operator experience and patient expectations. This everyday scientific evidence presented in indexed journals around the world with constantly updated. The aim of this review is to present and describe the characteristics of the scientific impact of journals in the field of Dentistry serving as a basis for better clinical decision making in our patients. The search for information was made according to index impact factor ISI Web of Science 2014.

KEY WORDS: impact factor, journal impact factor, portals for scientific journals, Medline, journal article.

\section{REFERENCIAS BIBLIOGRAFICAS}

Berhidi, A.; Szluka, P. \& Vasas, L. New bibliometric indicators. Is this the end of the impact factor era? Magy. Onkol., 53(2):115-25, 2009.

Brown, T. Journal quality metrics: options to consider other than impact factors. Am. J. Occup. Ther., 65(3):346-50, 2011.

Chang, C. L.; McAleer, M. \& Oxley, L. Coercive journal self citations, impact factor, Journal Influence and Article Influence. Math. Comput. Simul., 93:190-7, 2013.

Falagas, M. E.; Kouranos, V. D.; Arencibia-Jorge, R. \& Karageorgopoulos, D. Comparison of SCImago journal rank indicator with journal impact factor. FASEB J., 22(8):2623-8, 2008.

Fardi, A.; Kodonas, K.; Gogos, C. \& Economides, N. Top-cited articles in endodontic journals. J. Endod., 37(9):1183-90, 2011.

Feijoo, J. F.; Limeres, J.; Fernández-Varela, M., Ramos, I. \& Diz, P. The 100 most cited articles in dentistry. Clin. Oral Investig., 18(3):699-706, 2014.

Garfield, E. The history and meaning of the journal impact factor. JAMA, 295(1):90-3, 2006.

González-Pereira, B.; Guerrero-Bote, V. P. \& Moya-Anegón, F. A new approach to the metric of journals' scientific prestige: The SJR indicator. J. Informetr., 4(3):379-91, 2010.

Jalalzadeh, A. \& Sangari, M. The study scientific production visibility Iran University of Medical Sciences according to Matthew in journals indexed in the WoS at five year period. En: Proceedings of the Third National Conference on Research in the field of medical science. Babol, Nashr Ketabdar, 2011.

Kianifar, H.; Sadeghi, R. \& Zarifmahmoudi, L. Comparison Between
Impact Factor, Eigenfactor Metrics, and SCimago Journal Rank Indicator of Pediatric Neurology Journals. Acta Inform. Med., 22(2):103-6, 2014.

Kulasegarah, J. \& Fenton, J. E. Comparison of the h index with standard bibliometric indicators to rank influential otolaryngologists in Europe and North America. Eur. Arch. Otorhinolaryngol., 267(3):455-8, 2010.

Oosthuizen, J. C. \& Fenton, J. E. Alternatives to the impact factor. Surgeon, 12(5):239-43, 2014.

Owlia, P.; Vasei, M.; Goliaei, B. \& Nassiri, I. Normalized impact factor (NIF): an adjusted method for calculating the citation rate of biomedical journals. J. Biomed. Inform., 44(2):216-20, 2011.

Ramin, S. \& Sarraf Shirazi, A. Comparison between Impact factor, SCImago journal rank indicator and Eigenfactor score of nuclear medicine journals. Nucl. Med. Rev. Cent. East Eur., 15(2):132-6, 2012.

Sillet, A.; Katsahian, S.; Rangé, H.; Czernichow, S. \& Bouchard, P. The Eigenfactor ${ }^{\mathrm{TM}}$ Score in highly specific medical fields: the dental model. J. Dent. Res., 91(4):329-33, 2012.

Yaminfirooz, M.; Siamian, H.; Jahani, M. A. \& Yaminifirouz, M. Scientific production of Sports Science in Iran: A Scientometric Analysis. Acta Inform. Med., 22(3):195-8, 2014.

Dirección para Correspondencia:

Académico, Posgrado en Estomatología Universidad Peruana Cayetano Heredia

Lima

PERÚ

Email: marco.alarcon@upch.pe

Recibido: 03-10-2014

Aceptado: 09-02-2015 